\title{
Malathion induces anxiety in the male adult mouse
}

Goudarz Sadeghi Hashjin¹, Farhad Sadeghi Dizaj², Hadi Attaran², Mohammad Kazem Koohi ${ }^{1}$

${ }^{1}$ Faculty of Veterinary Medicine, University of Tehran, Tehran, Iran

${ }^{2}$ Faculty of Veterinary Medicine, Islamic Azad University - Tabriz Campus, Tabriz, Iran

Submitted: 12 November 2010

Accepted: 8 December 2011

Arch Med Sci 2013; 9, 2: 368-371

DOI: 10.5114 /aoms.2013.33174

Copyright (C 2013 Termedia \& Banach

\section{Abstract}

Introduction: The cholinergic nervous system and acetylcholine esterase are involved in chronic intoxication with organophosphorous insecticides. The present study aims to investigate the influence of the chronic toxicity of these chemicals on behaviors related to anxiety, using the elevated plus maze (EPM), in the male adult mouse.

Material and methods: Either water or $1 \%$ concentration of malathion was applied dermally to the male adult mice (10 s, once daily for 28 days) and, on day 29, the EPM test was done.

Results: Time spent in the open arms (TSOA) in intoxicated animals was decreased by over $50 \%$ compared to the controls $(p=0.047)$. In contrast, time spent in closed arms was significantly higher in the malathion-exposed mice $(p=0.025)$. Percentage of open arm entries (OAE) was slightly smaller in the malathion-treated group in comparison to the control animals. Percentage of closed arm entries (CAE) in the treated group was slightly higher than the value in the control animals.

Conclusions: The results showed that chronic toxicity of malathion may lead to an anxiety-like behavior in the animal model used in this study. It is difficult to extend these findings to clinical situations. However, more experimental work in different animal species as well as epidemiological studies in human subjects in this regard are highly recommended.

Key words: anxiety, elevated plus maze, malathion, mouse, organophosphates.

\section{Introduction}

Malathion is an organophosphate widely used as an insecticide in agriculture and in public health programs, causing risk to human health. Due to a wide range of usages in the agricultural sector, malathion is a highscale chemical in use all over the world. The estimated average annual total domestic usage of malathion in the USA is approximately 15 million pounds of malathion as an active ingredient [1]. Acute toxicity in rodents following exposure to organophosphate compounds has long been understood [2]. Repeated exposure to organophosphates has been shown to cause a decrease in muscarinic cholinergic receptors in the central and peripheral nervous system [3]. The mechanism of acute toxicity of the organophosphorus insecticides has been known for many years to be inhibition of the critical enzyme acetylcholinesterase (EC 3.1.1.7), with the resulting excess acetylcholine accumulation leading to symptoms of cholinergic excess [4]. Acute organophosphate poisoning is known to result in substantial behavioral abnormalities. Psychiatric manifestations of exposure in workers less substantially exposed to organophosphate compounds

\section{Corresponding author:} Goudarz Sadeghi Hashjin PhD Faculty of Veterinary Medicine University of Tehran P.O. Box 6453, Tehran 14155 Iran Phone: +9821611 17190 Fax: +9821669332 22 E-mail: gsadeghi@ut.ac.ir 
and showing no obvious signs of toxicity were investigated. The commercial sprayers but not the exposed farmers showed elevation of anxiety and lower plasma cholinesterase than control subjects. These findings were viewed as tentative until confirmed by additional studies, but they point to the possibility that organophosphate compounds may produce subtle defects in workers who do not show obvious toxicity [5]. Previous studies support the value of detailed clinical observations to screen for the neurotoxic potential of organophosphates and a general standard of more than $20 \%$ inhibition of brain cholinesterase activity for cholinergic neurotoxicity [6].

The elevated plus maze (EPM) is a simple instrument to analyze some behaviors of mice and rats. It was introduced by Pellow et al. first in 1985 for anxiety studies in the rat [7]. It consists of two crossing arms which make a plus-like configuration. One arm, surrounded by walls, is called the closed arm and the other, not surrounded by walls, is called the open arm. Rodents generally prefer to stay longer in the closed arms and are afraid of moving and staying on the open arms. Their movement on the open arms, especially on the remote one third of this part, is limited to searching the environment and some sort of curiosity as the height of the instrument is a kind of stress for them. Two years after Pellow, the EPM was also designed in smaller dimensions to be used for the mouse [8]. A recent work published by our group demonstrated a valuable finding in the mouse exposed to lead acetate, using the EPM; as suggested by the study, the exposure caused a kind of increased risky behavior in this species [9].

It is regrettable that not much attention has been paid to the behavioral influences of environmental pollutants using more developed experimental models. The aim of the present study was to investigate whether a widely used insecticide, malathion, may affect the basic anxiety status in the male adult mouse as an animal model.

\section{Material and methods}

\section{Animals and chemicals}

Twenty male adult Balb/c mice were obtained from Razi Vaccine and Serum Research Institute (Marand, Iran). Their weight range was 20-25 g and they were 8 weeks old when transferred to the lab. They were kept for 1 week to adapt to the new conditions. The mice were kept at room temperature with free access to commercial chow and tap water. Light and dark was supplied in $12 \mathrm{~h}$ cycles. Malathion 57\% EC was a product of Melli Agrochemical Company (Qazvin, Iran).

\section{Experimental protocol}

The protocol, including ethical points in case of animal care issues, was approved as an undergra- duate research project by the Research Council of the Islamic Azad University of Tabriz. Animals were divided into two groups of 10 each. The controls were exposed topically to saline solution from the whole tail area for $10 \mathrm{~s}$ once daily for 28 successive days. The treated group was exposed in the same manner to a $1 \%$ concentration (100 ppm) of malathion. On day 29, the animals were placed individually in the elevated plus maze (EPM) for 5 min (300 s). The area was kept silent and dark while the EPM was under a slight light emitted from a red $60 \mathrm{~W}$ lamp placed at a $50 \mathrm{~cm}$ distance from the top of the maze. Movements of the animals were recorded by a video camera (Canon MV690 E) connected to a computer (Asus Eee PC) and viewed by Windows Media Player 11. Time spent in open arms (TSOA), time spent in closed arms (TSCA), open arm entries (OAE) and closed arm entries (CAE) were determined and recorded.

\section{Statistical analysis}

Data were averaged in every experimental group and expressed as means \pm standard error of the means (SEM). Then, differences were evaluated by unpaired Student's $t$ test. A value of $p<0.05$ was taken as statistically significant.

\section{Results}

\section{Time spent in the open and closed arms}

Time spent in open arms in intoxicated animals was decreased by over $50 \%$ compared to the controls ( $p=0.047$, Figure 1 A). In contrast, TSCA was significantly higher in the malathion-exposed mice $(p=0.025$, Figure $1 \mathrm{~B})$.

\section{Open and closed arm entries}

Open arm entries in 5 min was slightly smaller in the malathion-treated group in comparison to the control animals (not statistically significant, Figure $1 \mathrm{C}$ ). Closed arm entries in the treated group was slightly higher than the value in the control animals (not significant, Figure $1 \mathrm{D}$ ).

\section{Discussion}

The present study showed that chronic toxicity with malathion may lead to a state of anxiety-like behavior in the male adult mouse. The experiments were done in ordinary conditions with no stress imposed on the animal; therefore, the influence was exerted on basic anxiety levels in rodents.

$M_{1}$ and $M_{2}$ receptor subtypes may be similarly regulated in response to subchronic exposure to organophosphates, including malathion [2]. $\mathrm{LD}_{50}$ values (median lethal dose for $50 \%$ of animals) values determined for many pesticides administered to adult and weanling male and female rats sug- 

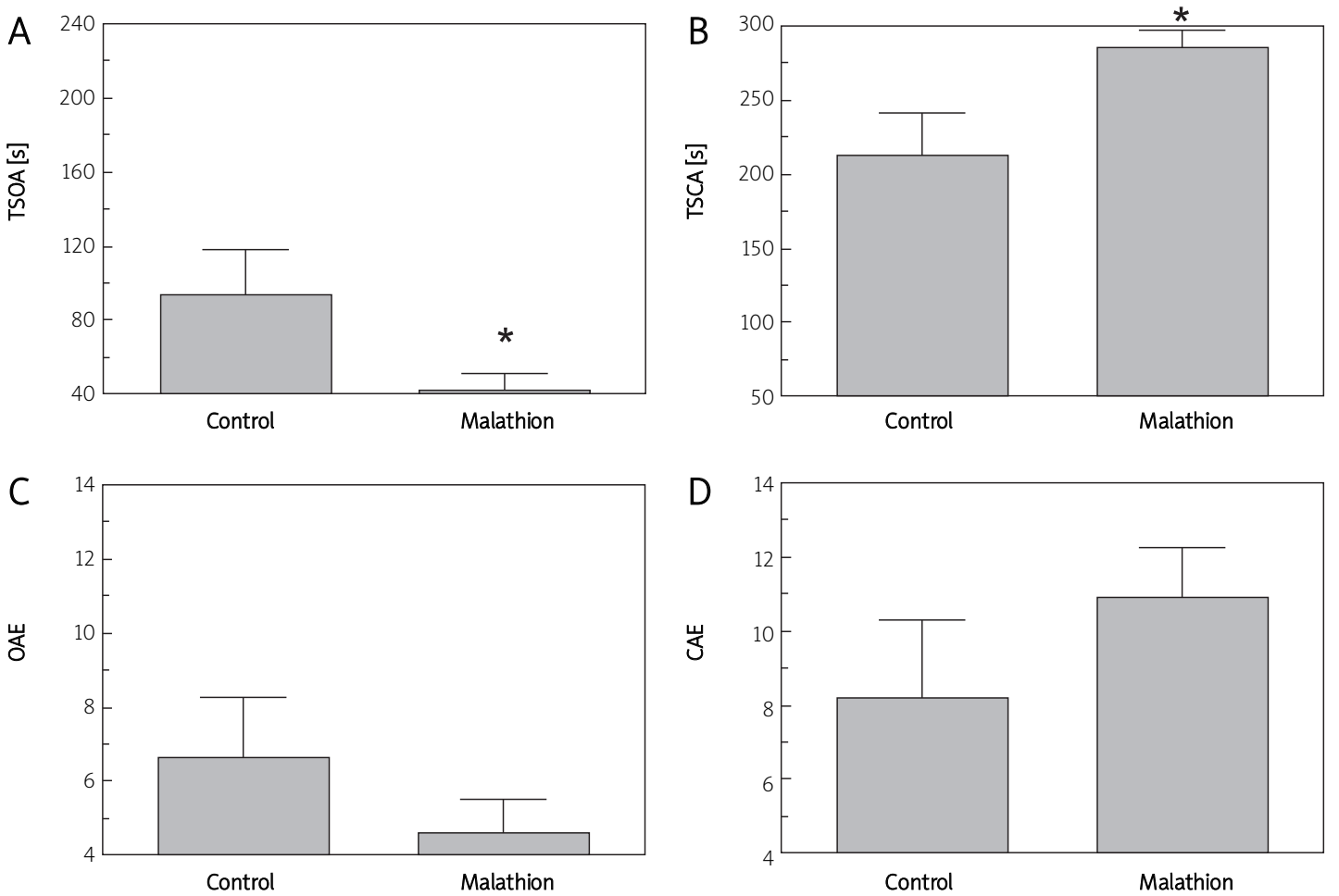

Figure 1. Effect of malathion on the behavior of the male adult mouse in EPM. Time spent in the open arms (TSOA, A), time spent in the closed arms (TSCA, B), open arm entries (OAE, C) and closed arm entries (CAE, D) are depicted in the figure. Malathion caused a significant decrease in TSOA and increased TSCA

${ }^{*} p<0.05$

gested that there were gender and age differences in the response of the animals to different agents [10]. Acute organophosphate intoxication in man is well known to result in substantial central nervous system dysfunction. However, relative resistance of higher nervous system functions to mild chronic organophosphate exposure has been suggested by existing results [11]. Malathion influences the reproductive function both by its cellular toxic action and by affecting the encephalic regulatory serotoninergic, besides acetylcholinergic, mechanism of the reproductive functions [12]. Malathion induces depressant-like behavior and oxidative damage to the brain of rodents [13]. Dichlorvos, another organophosphate agent, was shown to depress the open field behavior, ambulation, motor activity, and fine movements in the rat, with concordance of the open field behavioral changes with the levels of dopamine, norepinephrine, and serotonin in various regions of the brain [14]. This evidence is stressed only to note the relevance of behavioral abnormalities to organophosphate intoxication and it is not possible to make a connection between the existing findings and those of the current research work. However, peripheral effects cannot be ruled out in the present experimental conditions.

Considering some findings, which indicate the increase of brain serotonin by malathion, this mechanism may be the one which caused anxiety in our animal model. Serotoninergic neurotransmis- sion increase could be acting within the amygdala, enhancing anxiety [15]. It is suggested in individuals suffering predisposition to anxiety disorders to minimize the exposure to malathion by means of sanitary measures. Summarized in one sentence, the message is that long-term exposure to insecticides is likely to cause not only somatological but also behavioral disorders in humans. Our findings suggest an area warranting more systematic and definitive investigation, and the role of cholinergic brain transmission in anxiety disorders could be a very interesting research line.

\section{References}

1. EPA. Registration and eligibility decision (RED) for malathion. 2008. United States Environmental Protection Agency (EPA 738-R-06-030).

2. Gaines TB. The acute toxicity of pesticides to rats. Toxicol Appl Pharmacol 1960; 2: 88-99.

3. Fitzgerald BB, Costa LGM Modulation of $M_{1}$ and $M_{2}$ muscarinic receptor subtypes following repeated organophosphate exposure in rats. Toxicol Appl Pharmacol 1992; 117: 122-5.

4. Kardos SA, Sultatos LG. Interactions of the organophosphates paraoxon and methyl paraoxon with mouse brain acetylcholinesterase. Toxicol Sci 2000; 58: 118-26.

5. Levin HS, Rodnitzky RL, Mick DL. Anxiety associated with exposure to organophosphate compounds. Arch Gen Psychiatry 1976; 33: 225-8.

6. Sheets LP, Hamilton BF, Sangha K, Thyssen JH. Subchronic neurotoxicity screening studies with six organophosphate 
insecticides: an assessment of behavior and morphology relative to cholinesterase inhibition. Fundam Appl Toxicol 1997; 35: 101-19.

7. Pellow S, Chopin P, File SE, Briley M. Validation of open: closed arm entries in an elevated plus-maze as a measure of anxiety in the rat. J Neurosci Methods 1985; 14: 149-67.

8. Lister RG. The use of a plus-maze to measure anxiety in the mouse. Psychopharmacol (Berl) 1987; 92: 180-5.

9. Sadeghi-Hashjin G, Dehrouye M, Arab H, Mohammadyar L. Effect of chronic lead intoxication on risky behavior in mice. J Shaheed Sadoughi Uni Med Sci 2010; 18 (High Risk Behavior Conference Supplement 2010): 159-63.

10. Gaines TB, Linder RE. Acute toxicity of pesticides in adult and weanling rats. Fundam Appl Toxicol 1986; 7: 299-308.

11. Rodnitzky RL. Occupational exposure to organophosphate pesticides: a neurobehavioral study. Arch Environ Health 1975; 30: 98-103.

12. Uluitu M, Boca A, Petec G, Chis R, Catrinescu G. The influence of malathion on the brain serotonin and reproductive function in rats. Physiologie 1981; 18: 167-74.

13. Brocardo PS, Assini F, Franco JL, et al. Zinc attenuates malathion-induced depressant-like behavior and confers neuroprotection in the rat brain. Toxicol Sci 2007; 97: 140-8.

14. Ali SF, Chandra O, Hasan M. Effects of an organophosphate (dichlorvos) on open field behavior and locomotor activity: correlation with regional brain monoamine levels. Psychopharmacology (Berl) 1980; 68: 37-42.

15. Graeff FG, Zangrossi H Jr. The dual role of serotonin in defense and the mode of action of antidepressants on generalized anxiety and panic disorders. Cent Nerv Syst Agents Med Chem 2010; 10: 207-17. 